\title{
The Relationship of CSF and Plasma Cytokine Levels to Cerebral White Matter Injury in the Premature Newborn
}

\author{
VANESSA J. ELLISON, TESSA J. MOCATTA, CHRISTINE C. WINTERBOURN, \\ BRIAN A. DARLOW, JOSEPH J. VOLPE, AND TERRIE E. INDER
}

Neonatal Medicine [V.J.E., T.E.I.], Royal Women's and Royal Children's Hospitals, Melbourne, Victoria 3102, Australia, Department of Pathology [T.J.M., C.C.W.], Department of Pediatrics [B.A.D., T.E.I.], Christchurch School of Medicine and Health Sciences, Christchurch 8001, New Zealand, Department of Neurology [J.J.V.], Children's Hospital and Harvard Medical School, Boston, Massachusetts 02115, Murdoch Children's Research Institute and Howard Florey Institute [T.E.I.], University of Melbourne, Melbourne 3052, Australia

\begin{abstract}
Ischemia and systemic infection are implicated in the etiology of periventricular white matter injury, a major cause of adverse motor and cognitive outcome in preterm infants. Cytokines are signaling proteins that can be produced as part of the inflammatory response to both ischemia and infection. The aim of this study was to relate cerebrospinal fluid (CSF) concentrations of IL-6, IL-8, IL-10, tumor necrosis factor alpha (TNF- $\alpha$ ), and interferon gamma (IFN- $\gamma$ ) to magnetic resonance-defined white matter injury in preterm infants. Relationships between CSF and plasma cytokine concentrations were also examined. Preterm infants ( $\leq 32$ wk) and more mature infants from The Royal Women's Hospital, Melbourne, Australia, and Christchurch Women's Hospital, Christchurch, New Zealand, were eligible for study if they required a clinically indicated lumbar puncture. Plasma samples were obtained in a subgroup of Christchurch infants. Preterm infants underwent advanced quantitative volumetric magnetic resonance imaging using a 1.5-Tesla scanner at term equivalent. One hundred forty-six infants were enrolled and
\end{abstract}

\section{ABSTRACT}

190 CSF and 42 plasma samples obtained. There was no significant correlation between paired CSF and plasma concentrations for any cytokine. In comparing plasma and CSF concentrations, levels of IL-8 were significantly higher in CSF than plasma. Preterm infants with MRI-defined cerebral white matter injury had higher levels of IL-6, IL-10, and TNF- $\alpha$ in the CSF than infants without such injury. Plasma cytokine concentrations may not reflect CSF cytokine levels or inflammatory events within the brain. Elevated CSF levels of cytokines in infants with white matter injury suggest an altered inflammatory balance. (Pediatr Res 57: 282-286, 2005)
CSF, cerebrospinal fluid
IFN- $\boldsymbol{\gamma}$, interferon gamma
MR, magnetic resonance
TNF- $\boldsymbol{\alpha}$, tumor necrosis factor alpha
WMI, white matter injury

Abbreviations
White matter injury (WMI) is a major neuropathology in preterm infants associated with adverse motor and cognitive outcome. The introduction of MR imaging has led to an increased recognition of the more common diffuse noncystic WMI in preterm infants (1-6). The etiology of periventricular WMI is not yet clear, but both ischemia and/or systemic infection/inflammation appear to be important pathogenic pathways (7-16).

Cytokines are signaling proteins that are produced as part of the inflammatory response associated with ischemia and infec-

Received May 26, 2004; accepted July 19, 2004.

Correspondence: Terrie Inder, M.D., Murdoch Children's Research Institute and Howard Florey Institute, Royal Women's and Royal Children's Hospital, Flemington Rd, Parkville VIC 3052, Australia; e-mail: terrie.inder@rch.org.au

DOI: 10.1203/01.PDR.0000148286.53572.95 tion. In experimental animal models of ischemic perinatal cerebral injury, increased pro-inflammatory cytokines such as IL-6, IL-8, and TNF- $\alpha$ have been observed in the brain 0-24 $\mathrm{h}$ after insult $(17,18)$. In addition, several animal studies have demonstrated periventricular WMI in association with maternal intrauterine infection or endotoxin injection (19-23). In the human preterm infant, raised concentrations of the proinflammatory cytokines IL- $1 \beta$, IL-6, IL-18, and TNF- $\alpha$ in blood or amniotic fluid have been associated with abnormal cranial ultrasound appearances consistent with PVL and later impairment in neurodevelopmental outcome (24-29). Duggan et al. (30) demonstrated that elevated inflammatory markers including higher concentrations of TNF- $\alpha$, IL-1 $\beta$, IL-6, IL-10, and $\mathrm{CD}_{4} 5 \mathrm{RO}^{+} \mathrm{T}$ lymphocytes in the umbilical cord blood predicted cerebral lesions detected by MR imaging soon after 
delivery. Despite this finding, there is still a lack of data addressing the relationship between blood and CSF concentrations of cytokines during postnatal life in the premature infant in relation to cerebral injury, particularly WMI, as defined by MR imaging.

Thus, this study aimed to firstly determine whether CSF levels of the inflammatory cytokines IL-6, IL-8, IL-10, TNF- $\alpha$, and IFN- $\gamma$ were elevated in relation to MR-defined WMI in the preterm infant and secondly to examine relationships between the concentrations of these cytokines in the CSF and plasma.

\section{METHODS}

Subjects. Infants were eligible for this study if they required a clinically indicated lumbar puncture examination and were admitted to the special care nursery or the neonatal intensive care unit at Christchurch Women's Hospital, Christchurch, New Zealand (January 1999 to June 2001) and The Royal Women's Hospital, Melbourne, Australia (November 2001 to May 2002). The study population comprised three groups: 1) infants $>32 \mathrm{wk}$ gestation with suspected sepsis but without any CNS pathology; 2) infants with gestational age $<32$ wk with MR evidence of WMI at term; and 3) infants with gestational age $<32$ wk without WMI.

Ethics. Informed written parental consent was obtained for all study infants. The study was approved by The Royal Women's Hospital Research Advisory Committee, Melbourne, Australia, and the Canterbury Ethics Committee, Christchurch, New Zealand.

Sample collection. The samples were acquired at the time of a clinically indicated lumbar puncture. Clinical indications included concern of a risk of meningitis and covered all patients with proven sepsis (positive blood culture for all organisms excluding Staphylococcus epidermidis) or high risk for sepsis, including any of the following: a) C-reactive protein $>10 \mathrm{mg} / \mathrm{L}$, b) neutrophil count of immature band forms $>1.0 \times 10^{9} / \mathrm{L}, \mathrm{c}$ ) immature band neutrophils/total neutrophil ratio $>0.2$, and/or d) clinical evidence of septic shock. All consented infants had an additional $1 \mathrm{~mL}$ CSF collected under sterile conditions. Plasma samples were obtained within $24 \mathrm{~h}$ of collection of the CSF sample in a subgroup of infants from Christchurch.

Exclusion criteria. CSF samples were excluded from analysis if there was any evidence of meningeal inflammation by an elevated white cell count $(>5$ white blood cells and/or $>1$ polymorphonuclear cell correcting for CSF red cell count by 1:500) or positive CSF culture or antigen testing. Plasma samples were excluded from analysis if there was confirmed infection (positive culture from a normally sterile site and/or focal changes on chest x-ray).

Cytokine analysis. CSF and plasma samples were rapidly frozen at $-80^{\circ} \mathrm{C}$. Samples collected in Melbourne were transported frozen on dry ice to Christchurch for analysis. Cytokine analyses were performed by ELISA (Quantikine, R \& D Systems, Minneapolis, MN). Plates were coated overnight with mouse monoclonal anti-human IL-6, IL- 8 , IL-10, TNF- $\alpha$, or IFN- $\gamma$ as the capture antibody. Fifty-microliter aliquots of each sample were added to duplicate wells, followed by the respective biotinylated polyclonal detection antibody for each cytokine and then streptavidin horseradish peroxidase. Color was observed with TMB substrate (DAKO, Carpinteria, CA). Detection limits were as follows: IL-6, $4 \mathrm{pg} / \mathrm{mL}$; IL-8, $3 \mathrm{pg} / \mathrm{mL}$; IL-10, $5 \mathrm{pg} / \mathrm{mL}$; TNF- $\alpha, 7$ $\mathrm{pg} / \mathrm{mL}$; and IFN- $\gamma, 3 \mathrm{pg} / \mathrm{mL}$. Intra- and interassay variability was $\leq 5 \%$ and $10 \%$, respectively, for all cytokines.

MRI protocol. The preterm infants underwent advanced quantitative volumetric MRI scanning using a 1.5-Tesla MR scanner at term equivalent. The infants were fed, wrapped, and placed in a vacuum-extracted pillow. No sedation was used. For the acquisition of the primary MR data, two different imaging modes were applied—a three-dimensional Fourier Transform spoiled gradient recalled (SPGR) sequence $\left(1.5-\mathrm{mm}\right.$ coronal slices, flip angle $45^{\circ}$, repetition time $35 \mathrm{~ms}$, echo time $5 \mathrm{~ms}$, field of view $18 \mathrm{~cm}$, matrix $256 \times 256$ ) and a double-echo [proton density (PD) and T2-weighted] spin echo sequence (DE) (3-mm axial slices, repetition time $3000 \mathrm{~ms}$, echo times 36 and $162 \mathrm{~ms}$, field of view $18 \mathrm{~cm}$, matrix $256 \times 256$, interleaved acquisition).

MRI definition of cerebral WMI. All images were independently read by a qualified pediatric neuroradiologist blinded to the infant's clinical course. The images were assessed using a standardized scoring sheet (2). This scoring classified the presence of WMI as either 1) severe cerebral WMI consisting of cystic white matter injury and ventriculomegaly, consistent with diffuse loss of white matter volume; 2) moderate cerebral WMI with signal abnormality in periventricular WM and ventriculomegaly, but no cysts; 3) mild cerebral WMI with focal white matter signal abnormality but no cysts or definite ventriculomegaly; or 4) normal. The presence of any severity of WMI (mild, moderate, or severe) was classified as WMI for the analysis of the relationship of cytokines to WMI.

Data analysis. Data analysis was undertaken with SigmaStat and Sigmaplot (Jandel Scientific, Costa Madre, CA). Comparisons were made by KruskalWallis or ANOVA on rank and as appropriate followed by the Mann-Whitney $U$ test. The cytokine levels in paired CSF and plasma samples were compared by the Wilcoxon signed rank test, and the relationship between these samples was confirmed with analysis by Spearman rank order.

\section{RESULTS}

There were 146 infants enrolled with a total of $190 \mathrm{CSF}$ samples collected for cytokine analysis. Thirty four infants were excluded due to evidence of infection in the CSF with $>5$ WBC per 500 RBC, positive culture, or positive Gram's stain. The CSF samples from six infants $(n=7$ samples) were excluded because the infants did not undergo an MRI scan. Of the remaining 106 infants (111 CSF samples) that were eligible for analysis, 36 infants $(34 \%)$ were very preterm $(<32 \mathrm{wk}$ gestational age) and 70 infants $(65 \%)$ were $>32 \mathrm{wk}$ GA. Their characteristics are shown in Table 1 . Of the very preterm infants, 21 (66\%) had evidence of cerebral WMI: 8 cystic, 5 white matter signal abnormality, and 8 severe ventriculomegaly. Of the infants $>32 \mathrm{wk}$, only 15 infants underwent MR imaging with no WMI detected in any infant.

CSF results. Gestational age and birth weight were not significantly difference between the preterm infants with and without WMI (Table 1). However, it is important to note that the CSF samples obtained from the preterm infants with WMI were collected at a significantly later time after birth than the preterm infants without WMI. Figure 1 shows the relationship between the CSF cytokine levels and the day of sampling for all infants. On regression analysis of the data for the $>32 \mathrm{wk}$ infants (the only group for which there was a sufficient spread of samples), there were no significant relationships between time of sample and the concentration of any cytokine. This suggests differences in the time of sampling may not be a major confounder in comparing the infant groups.

Premature infants with evidence of cerebral WMI on MRI at term equivalent had significantly elevated levels of IL-6, IL-10,

Table 1. Characteristics of the study infants [median (range)]

\begin{tabular}{lcccc}
\hline & $<32$ wk preterm with WMI & $<32$ wk preterm without WMI & $>32$ wk GA & $p$ Value $(t$ test) (preterm with and without WMI) \\
\hline No. of infants & 21 & 15 & 70 & 70 \\
No. of CSF samples & 25 & 16 & $39(37-40)$ & 0.129 \\
Gestational age (wk) & $26(24-28)$ & $27.5(25-31.5)$ & $3220(2660-3560)$ & 0.456 \\
Birth weight (g) & $857(600-1133)$ & $820(687-1597.5)$ & $2(1-3)$ & 0.011 \\
Day of sample & $19(18-27)$ & $5(2-13.5)$ & \\
\hline
\end{tabular}




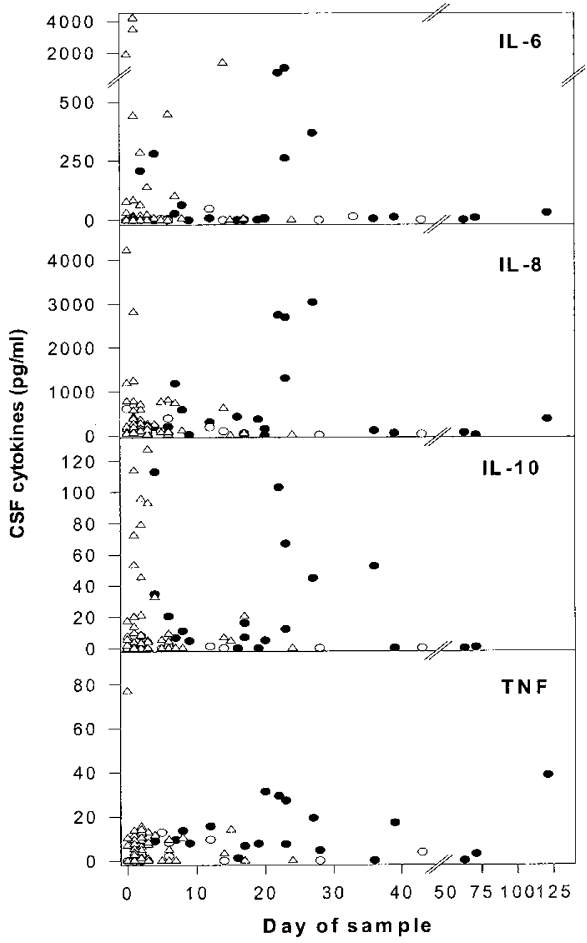

Figure 1. The relationship of CSF cytokine levels to the day of sampling. Closed O, infants $<32$ weeks gestation with WMI; Open O, infants $<32$ weeks gestation without WMI; Open triangle, infants of $>32$ weeks gestation.

and TNF- $\alpha$ in their CSF in comparison to premature infants without WMI. (Table 2, Fig. 2). Although no significant difference in the level of IFN- $\gamma$ was detected, it is noteworthy that only 2 of the 40 CSF samples had concentrations above the detection limit (Table 2).

There was no significant difference in the CSF levels of IL-6, IL-8, TNF- $\alpha$, and IFN- $\gamma$ between the very preterm infants without WMI and the $>32$ wk GA group. However, CSF IL-10 levels were significantly higher in the more mature infants $(p=0.008$, Table 2).

Relationships between cytokine concentrations in CSF and plasma. Of the $111 \mathrm{CSF}$ samples collected, 42 (38\%) had paired plasma samples. Of these, 13 were from preterm infants $<32$ wk gestation and included two additional samples from infants who did not have an MRI scan. The median day of sampling was $3 \mathrm{~d}$, interquartile range (IQR) d 1-6.

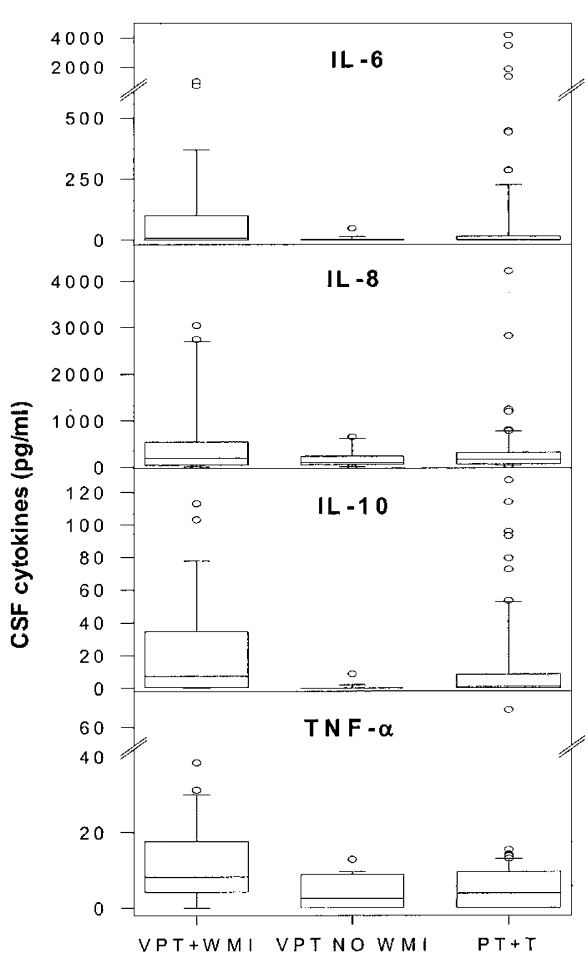

Figure 2. Boxplot (Median with 25th/75th centiles, error bars 10th/90th centile and outliers) of CSF cytokines in relation to preterm infants with WMI, preterm infants without WMI and all preterm and term infants combined.

With all preterm and term infants included, IL-10 and TNF- $\alpha$ concentrations were significantly higher in plasma than CSF (Table 3). There was no difference between plasma and CSF levels of IL-6. In contrast, IL-8 concentrations were significantly higher in CSF. There were no significant correlation between CSF and plasma concentration for any of the cytokines, although trends toward a correlation were strongest for IL-6 and TNF- $\alpha$.

In the infants $\leq 32$ wk gestation $(n=13)$, IL- 8 concentrations remained significantly higher in CSF than plasma (medians 131.3 and $13.9 \mathrm{pg} / \mathrm{mL}$ respectively, $p=0.003$ ). For $\mathrm{IL}-10(p=0.718)$ and TNF- $\alpha(p=0.268)$ there was no longer a difference between plasma and CSF, but CSF concentrations of IL-6 (median 0) were significantly lower than in plasma $(15.3 \mathrm{pg} / \mathrm{mL}, p=0.022$, paired $t$ test Wilcoxon signed rank). No significant correlations were seen between CSF and plasma

Table 2. Median (interquartile range) cytokine concentrations in CSF

\begin{tabular}{|c|c|c|c|c|c|}
\hline & $<32$ wk preterm with $\mathrm{WMI}$ & $<32$ wk preterm without WMI & $>32$ wk GA & $\begin{array}{l}p \text { value } \\
\text { (ANOVA) }\end{array}$ & $\begin{array}{c}p \text { Value ( } t \text { test }) \text { preterm with and } \\
\text { without WMI }\end{array}$ \\
\hline IL-6 (pg/mL) & $8.0(0.0-99.7)$ & $0(0-2.7)$ & $3.1(0-13.8)$ & 0.025 & 0.012 \\
\hline Number of cases & $n=25$ & $n=15$ & $n=69$ & & \\
\hline IL-8 (pg/mL) & $\begin{array}{c}199(43.5-543.8) \\
n=23\end{array}$ & $\begin{array}{c}95(54.3-232) \\
n=15\end{array}$ & $\begin{array}{c}153(63.6-314) \\
n=68\end{array}$ & NS & NS \\
\hline IL-10 (pg/ml) & $\begin{array}{l}7.4(0.6-34.7) \\
n=22\end{array}$ & $\begin{array}{l}0(0-0) \\
\quad n=14\end{array}$ & $\begin{array}{c}0.9(0-8.5) \\
n=66\end{array}$ & $<0.001$ & $<0.0001$ \\
\hline TNF- $\alpha(\mathrm{pg} / \mathrm{mL})$ & $\begin{array}{c}8.2(4.2-17.6) \\
n=21\end{array}$ & $\begin{array}{l}2.5(0-8.8) \\
\quad n=15\end{array}$ & $\begin{array}{l}3.8(0-9.5) \\
n=66\end{array}$ & 0.011 & 0.045 \\
\hline $\mathrm{IFN}-\gamma(\mathrm{pg} / \mathrm{mL})$ & $\begin{array}{c}0.0(0-0) \\
n=7\end{array}$ & $\begin{array}{l}0(0-0) \\
\quad n=11\end{array}$ & $\begin{array}{l}0(0-0) \\
\quad n=22\end{array}$ & NS & NS \\
\hline
\end{tabular}


Table 3. Cytokine concentrations in paired CSF and plasma samples

\begin{tabular}{|c|c|c|c|c|}
\hline & $\mathrm{CSF}$ & Plasma & Paired $t$ test signed rank $p$ & Correlation coefficient* \\
\hline IL-6 (pg/mL) & $n=37$ & $n=37$ & 0.080 & 0.31 \\
\hline Median & 0 & 17.7 & & $p=0.06$ \\
\hline IQR & $0-5$ & $10-40$ & & \\
\hline IL-8 (pg/mL) & $n=38$ & $n=38$ & $<0.0001$ & 0.24 \\
\hline Median & 167.2 & 22.0 & & $p=0.15$ \\
\hline IQR & $69-389$ & $2-101$ & & \\
\hline Median & 0 & 3.0 & & $p=0.62$ \\
\hline IQR & $0-5$ & $0-30$ & & \\
\hline$<$ Detection limit & $74 \%$ & $51 \%$ & & \\
\hline TNF- $\alpha(\mathrm{pg} / \mathrm{mL})$ & $n=27$ & $n=27$ & 0.010 & 0.35 \\
\hline Median & 1.1 & 9.2 & & $p=0.08$ \\
\hline IQR & $0-6$ & $0-28$ & & \\
\hline
\end{tabular}

* Spearman rank order correlation, values below detection limit set at zero.

concentration for any of the cytokines (range correlation coefficients $0.21-0.01, p>0.1$ for all measures).

\section{DISCUSSION}

In this cohort study we have demonstrated a lack of any correlation between paired CSF and plasma concentrations for any of the cytokines that we examined. This may indicate a significant limitation of plasma samples in reflecting the cerebral inflammatory response. Additionally, there was variation in whether plasma or CSF was the site of the highest cytokine level for each cytokine. For all infant groups, IL-10 and TNF$\alpha$ were higher in plasma than CSF samples, contrasting with the concentrations of IL-8 that were significantly higher in the CSF, including in the subgroup of $<32$ wk gestation infants. These findings have implications regarding the potential origin of an inflammatory response within the CNS and suggests that some components, at least, may be localized within the CSF rather than resulting from the dissemination of cytokines via the blood stream. There was a notable difference between IL-6 and IL-8 with IL- 6 being often undetectable in the matched blood-CSF in our samples. There may be variability in the timing as well as the tissue localization rendering variation between blood and CSF levels. This would require more serial sampling of both CSF and blood cytokine levels for clarification. Finally, we have demonstrated higher CSF concentrations of IL-6, IL-10, and TNF- $\alpha$ in preterm infants subsequently shown to have cerebral WMI by MR scanning at term equivalent, compared with those without WMI. These changes may reflect an altered inflammatory response within the CNS in preterm infants who develop cerebral WMI. In contrast, in comparing the preterm infants without WMI and infants of $>32 \mathrm{wk}$ gestation the only difference in the concentration of CSF cytokines was in the level of IL-10, which was higher in the CSF of more mature infants.

There are a number of limitations to our study. Firstly, the preterm infants with cerebral WMI had their samples taken significantly later than the preterm infants without cerebral WMI and the $>32 \mathrm{wk}$ GA infants. This raises the possibility that an increase in cytokine levels with age could explain their higher values. However, no relationship was seen between day of sampling and concentrations of any of the cytokines in the $>32$ wk gestation group, making this explanation unlikely. Secondly, very few infants had more than one CSF sample, meaning that we were not able to define the evolution or pattern of the inflammatory response over time. And lastly, because the number of infants with WMI was small we were unable to determine whether the inflammatory response differed according to the severity of that injury.

A previously published study examining the cytokine response in CSF after birth asphyxia demonstrated that the pro-inflammatory cytokines IL-6 and IL- 8 were increased in the CSF of asphyxiated infants, but the levels of IL1- $\beta$ and IL-10 were not (31). The authors concluded that the inflammatory system played a pivotal role in the pathogenesis of cerebral injury after hypoxia-ischemia. IL- 6 and TNF- $\alpha$ have also been directly demonstrated on neuropathology within the cerebral lesions of PVL in human infants $(25,32)$. Our study is the first to have analyzed CSF cytokine levels in relation to MR-defined cerebral WMI and our results are further direct evidence for the association of an inflammatory process in the CNS and subsequent injury.

A role for infection/inflammation in the pathogenesis of PVL and cerebral palsy (CP) in premature infants has been suggested from studies showing increased rates of PVL and/or $\mathrm{CP}$ in association with maternal/placental/fetal infection $(7,13,33)$, with elevated levels of IL- 6 and IL- $1 \beta$ in amniotic fluid (25) and with elevated levels of IL-6 in cord blood (24). Nelson et al. (34) recently examined the relationship of blood cytokine concentrations from archived neonatal blood spots to the development of cerebral palsy in a cohort of infants who were born before $32 \mathrm{wk}$ gestational age. This study found that premature infants with ultrasound abnormalities averaged higher blood cytokine levels, in contrast with most surviving preterm infants who demonstrated low blood levels of inflammatory cytokines during the first days of life. In comparison to the current study, these samples were taken at a mean age (SD) of 2.4 (3.8) $\mathrm{d}$ for the cerebral palsy group and 2.4 (3.5) $\mathrm{d}$ for the control infants. The secondary aim of our study may assist in 
the understanding of a potential source of any conflicting results with regard to cerebral injury in relation to blood cytokine measurements in the preterm infant as we document a very poor correlation between blood and CSF cytokines in preterm infants. In our study, CSF cytokines were significantly elevated in preterm infants with WMI, but CSF levels did not correlate well with blood levels.

IL-10 is an anti-inflammatory cytokine produced by $\mathrm{CD} 4^{+} \mathrm{T}$ cells/B cells, monocytes, and, in utero, the placenta. Functionally, it down-regulates the pro-inflammatory cascade through inhibition of the production of pro-inflammatory mediators by macrophages (35-38). Our study shows an elevation of this anti-inflammatory cytokine in the CSF of premature infants with WMI. In contrast to our findings, data from the lungs of preterm infants with chronic lung disease have demonstrated more severe lung injury is associated with absent or irregular secretion of this down-regulatory cytokine in tracheobronchial aspirate fluid (39). However, others have reported that very preterm infants (40) and preterm baboons (41) are capable of producing IL-10 in bronchoalveolar fluids in response to injury. The elevation in IL-10 in the preterm infant with WMI is more consistent with these latter studies. What the role of IL-10 is in the development or prevention of injury remains to be determined.

In conclusion, this is the first study to examine the relationship of CSF to blood cytokine levels as well as the relationship of CSF cytokine levels to MRI-defined cerebral WMI. These preliminary data suggest that cerebral WMI in the preterm infant may be associated with an altered balance of inflammation within the CNS and that this inflammatory response involving cytokine activation may be localized within the CSF and not part of a more widespread systemic response.

\section{REFERENCES}

1. Cioni G, Bertuccelli B, Boldrini A, Canapicchi R, Fazzi B, Guzzetta A, Mercuri E 2000 Correlation between visual function, neurodevelopmental outcome, and magnetic resonance imaging findings in infants with periventricular leucomalacia. Arch Dis Child Fetal Neonatal Ed 82:F134-F140

2. Inder TE, Wells SJ, Mogridge N, Spencer C, Volpe JJ 2003 Defining the nature of the cerebral abnormalities in the premature infant: a qualitative magnetic resonance imaging study. J Pediatr 143:171-179

3. Barkovich AJ, Kjos BO, Jackson DE Jr, Norman D 1988 Normal maturation of the neonatal and infant brain: MR imaging at $1.5 \mathrm{~T}^{1}$. Radiology 166:173-180

4. Paneth N, Rudelli R, Monte W, Rodriguez E, Pinto J, Kairam R, Kazam E 1990 White matter necrosis in very low birth weight infants: neuropathologic and ultrasonographic findings in infants surviving six days or longer. J Pediatr 116:975-984

5. Huppi PS 2002 Advances in postnatal neuroimaging: relevance to pathogenesis and treatment of brain injury. Clin Perinatol 29:827-856

6. Volpe JJ 2001 Neurology of the Newborn. WB Saunders, Philadelphia

7. Leviton A, Gilles F 1996 Ventriculomegaly, delayed myelination, white matter hypoplasia, and "periventricular" leukomalacia: how are they related? Pediatr Neurol $15: 127-136$

8. Perlman JM, Risser R, Broyles RS 1996 Bilateral cystic periventricular leukomalacia in the premature infant: associated risk factors. Pediatrics 97:822-827

9. Zupan V, Gonzalez P, Lacaze-Masmonteil T, Boithias C, d'Allest AM, Dehan M, Gabilan JC 1996 Periventricular leukomalacia: risk factors revisited. Dev Med Child Neurol 38:1061-1067

10. de Vries LS, Regev R, Dubowitz LM, Whitelaw A, Aber VR 1988 Perinatal risk factors for the development of extensive cystic leukomalacia. Am J Dis Child 142:732-735

11. Back SA, Volpe JJ 1997 Cellular and molecular pathogenesis of periventricular white matter injury. MRDD Res Rev 3:96-107

12. Volpe JJ 2001 Neurobiology of periventricular leukomalacia in the premature infant. Pediatr Res 50:553-562
13. Kadhim H, Sebire G 2002 Immune mechanisms in the pathogenesis of cerebral palsy: implication of proinflammatory cytokines and T lymphocytes. Eur J Paediatr Neurol $6: 139-142$

14. Kadhim H, Tabarki B, De Prez C, Sebire G 2003 Cytokine immunoreactivity in cortical and subcortical neurons in periventricular leukomalacia: are cytokines implicated in neuronal dysfunction in cerebral palsy? Acta Neuropathol (Berl) 105:209216

15. Kadhim H, Tabarki B, Verellen G, De Prez C, Rona AM, Sebire G 2001 Inflammatory cytokines in the pathogenesis of periventricular leukomalacia. Neurology 56: $1278-1284$

16. O'Shea TM, Klinepeter KL, Meis PJ, Dillard RG 1998 Intrauterine infection and the risk of cerebral palsy in very low-birthweight infants. Pediatr Perinat Epidemiol $12: 72-83$

17. Hudome S, Palmer C, Roberts RL, Mauger D, Housman C, Towfighi J 1997 The role of neutrophils in the production of hypoxic-ischemic brain injury in the neonatal rat. Pediatr Res 41:607-616

18. Savman K, Blennow M, Hagberg H, Tarkowski E, Thoresen M, Whitelaw A 2002 Cytokine response in cerebrospinal fluid from preterm infants with posthaemorrhagic ventricular dilatation. Acta Paediatr 91:1357-1363

19. Gilles FH, Leviton A, Kerr CS 1976 Susceptibility of the neonatal feline telencephalic white matter to a lipopolysaccharide. J Neurol Sci 27:183-191

20. Yoon BH, Kim CJ, Romero R, Jun JK, Park KH, Choi ST, Chi JG 1997 Experimentally induced intrauterine infection causes fetal brain white matter lesions in rabbits. Am J Obstet Gynecol 177:797-802

21. Young RS, Yagel SK, Towfighi J 1983 Systemic and neuropathologic effects of E. coli endotoxin in neonatal dogs. Pediatr Res 17:349-353

22. Ando M, Takashima S, Mito T 1988 Endotoxin, cerebral blood flow, amino acids and brain damage in young rabbits. Brain Dev 10:365-370

23. Mallard C, Welin A-K, Peebles D, Hagberg H, Kjellmer I 2003 White matter injury following systemic endotoxemia or asphyxia in the fetal sheep. Neurochem Res 28:215-223

24. Yoon BH, Romero R, Yang SH, Jun JK, Kim IO, Choi JH, Syn HC 1996 Interleukin-6 concentrations in umbilical cord plasma are elevated in neonates with white matter lesions associated with periventricular leukomalacia. Am J Obstet Gynecol 174:1433-1440

25. Yoon BH, Jun JK, Romero R, Park KH, Gomez R, Choi JH, Kim IO 1997 Amniotic fluid inflammatory cytokines (interleukin-6, interleukin- $\beta$, and tumor necrosis factor- $\alpha$ ), neonatal brain white matter lesions, and cerebral palsy. Am J Obstet Gynecol 177:19-26

26. Adinolfi M 1993 Infectious diseases in pregnancy, cytokines and neurological impairment: an hypothesis. Develop Med Child Neurol 35:549-558

27. Baud O, Emilie D, Pelletier E, Lacaze-Masmonteil T, Zupan V, Fernandez H, Dehan M, Frydman R, Ville Y 1999 Amniotic fluid concentrations of interleukin-1beta, interleukin-6 and TNF-alpha in chorioamnionitis before 32 weeks of gestation: histological associations and neonatal outcome. Br J Obstet Gynaecol 106:72-77

28. Heep A, Behrendt D, Nitsch P, Fimmers R, Bartmann P, Dembinski J 2003 Increased serum levels of interleukin 6 are associated with severe intraventricular haemorrhage in extremely premature infants. Arch Dis Child Fetal Neonatal Ed 88:F501-F504

29. Minagawa K, Tsuji Y, Ueda H, Koyama K, Tanizawa T, Okamura H, HashimotoTamaoki T 2002 Possible correlation between high levels of IL-18 in the cord blood of pre-term infants and neonatal development of periventricular leukomalacia and cerebral palsy. Cytokine 17:164-170

30. Duggan PJ, Maalouf EF, Watts TL, Sullivan MH, Counsell SJ, Allsop J, Al-Nakib L, Rutherford MA, Battin M, Roberts I, Edwards AD 2001 Intrauterine T-cell activation and increased proinflammatory cytokine concentrations in preterm infants with cerebral lesions. Lancet 358:1699-1700

31. Savman K, Blennow M, Gustafson K, Tarkowski E, Hagberg H 1998 Cytokine response in cerebrospinal fluid after birth asphyxia. Pediatr Res 43:746-751

32. Deguchi K, Mizuguchi M, Takashima S 1996 Immunohistochemical expression of tumor necrosis factor $\alpha$ in neonatal leukomalacia. Pediatr Neurol 14:13-16

33. Yoon BH, Park CW, Chaiworapongsa T 2003 Intrauterine infection and the development of cerebral palsy. BJOG. 110:124-127

34. Nelson KB, Grether JK, Dambrosia JM, Walsh E, Kohler S, Satyanarayana G, Nelson PG, Dickens BF, Phillips TM 2003 Neonatal cytokines and cerebral palsy in very preterm infants. Pediatr Res 53:600-607

35. Moore KW, de Waal Malefyt R, Coffman RL, O'Garra A 2001 Interleukin-10 and the interleukin-10 receptor. Annu Rev Immunol 19:683-765

36. Marchant A, Bruyns C, Vandenabeele P, Ducarme M, Gerard C, Delvaux A, De Groote D, Abramowicz D, Velu T, Goldman M 1994 Interleukin-10 controls interferon-gamma and tumor necrosis factor production during experimental endotoxemia. Eur J Immunol 24:1167-1171

37. de Waal Malefyt R, Yssel H, Roncarolo MG, Spits H, de Vries JE 1992 Interleukin10. Curr Opin Immunol 4:314-320

38. Howard M, O'Garra A 1992 Biological properties of interleukin 10. Immunol Today 13:198-200

39. Jones CA, Cayabyab RG, Kwong KY, Stotts C, Wong B, Hamdan H, Minoo P, deLemos RA 1996 Undetectable interleukin (IL)-10 and persistent IL-8 expression early in hyaline membrane disease: a possible developmental basis for the predisposition to chronic lung inflammation in preterm newborns. Pediatr Res 39:966-975

40. McColm JR, Stenson BJ, Biermasz N, McIntosh N 2000 Measurement of interleukin 10 in bronchoalveolar lavage from preterm ventilated infants. Arch Dis Child Fetal Neonatal Ed 82:F156-159

41. Coalson JJ, Winter VT, Siler-Khodr T, Yoder BA 1999 Neonatal chronic lung disease in extremely immature baboons. Am J Respir Crit Care Med 160:1333-1346 